\title{
Inserción velamentosa de cordón y desaceleración variable en el monitoreo cardiaco fetal
}

\author{
Velamentous cord insertion and variable deceleration of fetal heart rate monitoring \\ Jorge Díaz ${ }^{1}$, Jorge Salvador ${ }^{1}$, Jaime Cok $^{2}$, Christian Zambrano ${ }^{3}$, Milagros Romero ${ }^{4}$. \\ RESUMEN
}

La inserción velamentosa de cordón es una patología inusual de la inserción de cordón, el cual carece de gelatina de Wharton. Presentamos el caso de una paciente que ingresó con diagnóstico presuntivo de corioamnionitis que presentó desaceleración variable (DIP III) durante el monitoreo fetal. La paciente fue operada sin estar en labor de parto y con membranas integras; el diagnóstico post cesárea fue inserción velamentosa de cordón.

PALABRAS CLAVE: Cordón umbilical, diagnóstico prenatal, monitoreo fetal. (Fuente: DeCS BIREME).

\section{SUMMARY}

Velamentous cord insertion is an unusual condition, Wharton's gelly protection is absent in this entity. We report a patient admitted with a presumptive diagnosis of chorioamnionitis that presented variable deceleration of fetal heart rate. A cesarean section was performed despite of not being in labor and having intact membranes. A velamentous cord insertion was confirmed.

KEYWORDS: Umbilical cord, velamentous cord insertion, variable deceleration. (Source: MeSH NLM).

\section{INTRODUCCIÓN}

La inserción velamentosa de cordón (IVC) se presenta en $1 \%$ de los embarazos simples y consiste en la inserción de los vasos fetales en las membranas, recorren cierta distancia entre amnios y corión antes de alcanzar la superficie placentaria. Ocurre en $10 \%$ de embarazos múltiples y es más frecuente en gestaciones con arteria umbilical única. Esta inserción anómala sería explicada por la teoría del trofotropismo, es decir ocurriría una inicial inserción normal pero al buscar la placenta el sito de mayor irrigación del miometrio se produciría la inserción velamentosa (1).

Cuando se asocia a vasa previa produce alta mortalidad, al romperse las membranas durante la labor de parto, además por carecer de gelatina de Warthon los vasos anómalos se laceran y hay hemorragia fetal. También puede ocurrir compresión de cordón, trombosis, retardo de crecimiento intrauterino, prematuridad, anomalías congénitas, placenta retenida y muerte fetal (1-3).

\footnotetext{
Ginecólogo Obstetra. Hospital Nacional Cayetano Heredia. Profesor Principal, Facultad de Medicina Alberto Hurtado. Universidad Peruana Cayetano Heredia, Lima, Perú.

2 Patólogo. Hospital Nacional Cayetano Heredia. Profesor Asociado, Facultad de Medicina Alberto Hurtado. Universidad Peruana Cayetano Heredia, Lima, Perú.

3 Ginecólogo Obstetra. Hospital Nacional Cayetano Heredia. Lima, Perú.

4 Obstetriz. Especialista en Monitoreo Fetal. Hospital Nacional Cayetano Heredia. Lima, Perú.
} 
Antes del uso de ultrasonido se tenía que reunir criterios clínicos y de laboratorio, según Naftolin y Mishel: sangrado vaginal acompañado de irregularidades del latido fetal, determinación del origen fetal de la sangre, palpación de vasa previa. Se consideraba también, la visualización de vasa previa con amnioscopio (4). Usando ultrasonido convencional complementado con ultrasonido Doppler y combinando vía abdominal y transvaginal se puede identificar la inserción de cordón tanto normal como patológica $(2,5)$.

Reportamos un caso sin diagnóstico pre natal de inserción velamentosa, que presentó cambios en el latido cardíaco fetal sin rotura de membranas, cuya detección permitió obtener un recién nacido en buenas condiciones. Se trata de un diagnóstico poco frecuente que permitió intervenir antes de la ruptura de membranas que hubiera provocado muerte fetal

\section{Caso clínico}

Paciente de 33 años, con 31 semanas de embarazo, transferida del Hospital Municipal con diagnóstico de posible pielonefritis vs corioamnionitis. Refería pérdida de líquido desde 3 días antes. Al examen clínico se encontró, pulso 88 por minuto, frecuencia respiratoria 20 por min, Temperatura $37^{\circ} \mathrm{C}$. Altura Uterina: $28 \mathrm{~cm}$. útero con tono normal. Latidos Fetales: 148 por minuto, Puño Percusión Lumbar derecho positivo. El examen ginecológico mostró, Dilatación
$=0$, Borramiento $=0$, Membrana: No evaluable, pelvis ginecoide. Se inició antibióticoterapia con Clindamicina $900 \mathrm{mg}$ EV c/8h y Ceftriaxona $2 \mathrm{~g} \mathrm{EV}$ $\mathrm{c} / 24 \mathrm{~h}$.

La paciente traía exámenes de laboratorio que mostraba un hemograma con 22500 leucocitos con 8 bastones y un examen de orina con leucocituria de 8-10 leucocitos por campo y nitritos negativo. Ultrasonido con perfil biofísico 10/10.

El día del ingreso se encontró un perfil biofísico ecográfico 8/8 y NST reactivo; hemograma con 9 900 leucocitos $\mathrm{x} \mathrm{mm}^{3}$. Al día siguiente se conoció el resultado de la amniocentesis: líquido meconial turbio, leucocitos: 1 x campo, Glucosa: $91 \mathrm{mg} / \mathrm{dl}$.

La paciente evolucionó afebril, 17,5 horas después se detectó durante el monitoreo electrónico fetal una desaceleración abrupta en la línea de base del latido cardiaco fetal, de más de 15 latidos, durante más de 15 segundos (DIP III o DIP variable), siendo sometida a cesárea, naciendo un varón de 2750 g, de 32 semanas por Capurro. Se encontró inserción velamentosa de cordón. El cultivo de líquido amniótico y de membranas tomado durante la cesárea, fue negativo.

El informe de anatomía patológica fue "Cordón umbilical de inserción velamentosa. Cordón y membranas sin alteraciones".

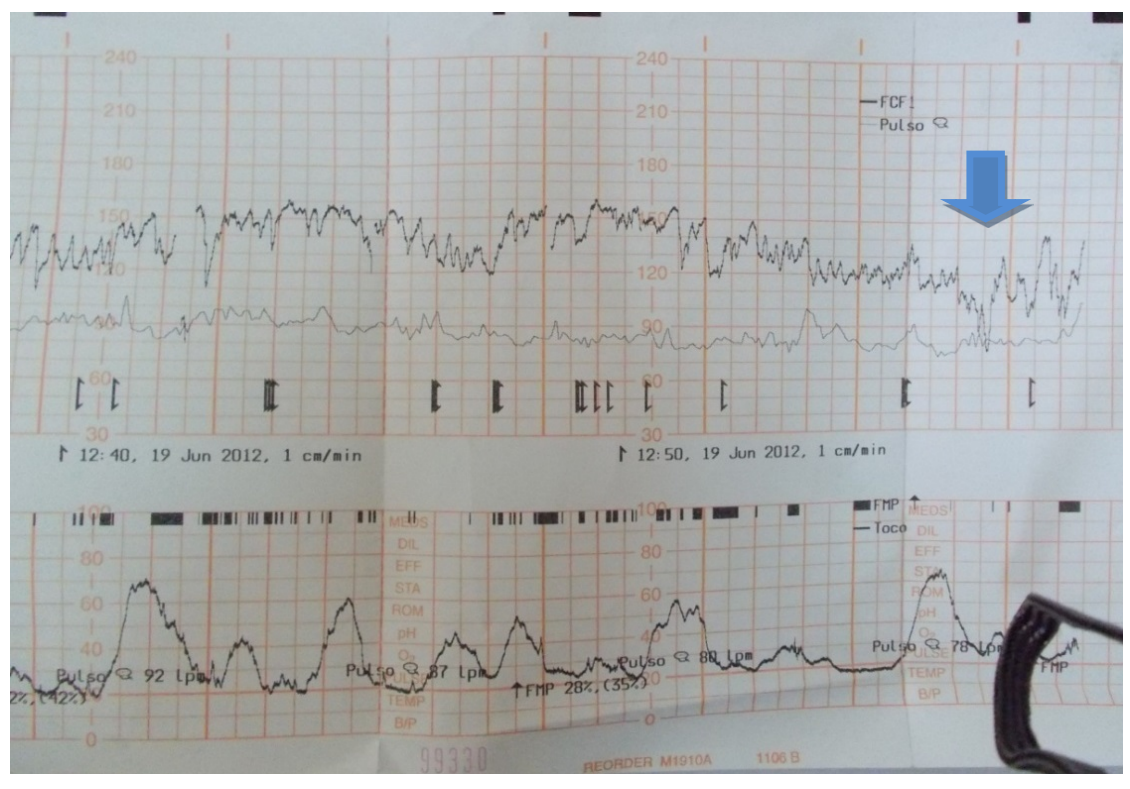

Figura 1. DIP VARIABLE. Trazado de monitoreo fetal. Disminución abrupta del latido cardíaco fetal de más de 15 latidos por más de 15 segundos (Flecha). 


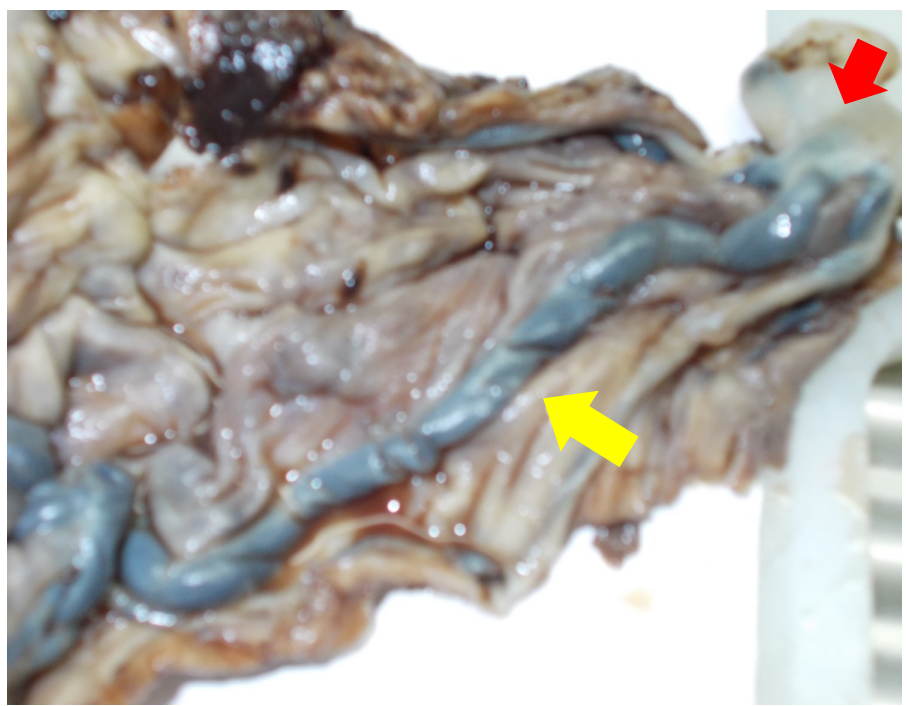

Figura 2. Inserción velamentosa. Cordón umbilical (flecha roja). Vasos anómalos (flecha amarilla).

\section{DISCUSIÓN}

A la paciente le realizaron dos ecografías durante la gestación, en diferentes instituciones; en ninguna hubo sospecha de inserción velamentosa de cordón. Se recomienda la búsqueda de anomalías de inserción de cordón en el segundo trimestre; Sepúlveda y col (5) combinando ultrasonido (US) 2D y Doppler abdominal y transvaginal, reportaron que de 8 casos de diagnóstico prenatal de IVC, 7 se verificaron en la anatomía patológica y uno correspondió a inserción marginal. Sin embargo, el American College of Radiology (ACR), The American Institute of Ultrasound in Medicine (AIUM) y American College of Obstetricians and Gynecologists (ACOG) no recomiendan en forma específica la evaluación de la inserción placentaria (6).

El trazado del monitoreo fetal mostró una desaceleración variable descrita en el caso clínico de acuerdo a las definiciones de National Institute of Child Health and Human Development (7), de cerca de 40 segundos lo que motivó el término de la gestación, en el contexto de una paciente con historia de fiebre y posibilidad de corioamnionitis. Clark (4), reportó un caso similar al nuestro con membranas íntegras sin labor de parto, en el que se había iniciado inducción con oxitocina 15 minutos antes. Hasegawa y col (8), en un estudio de casos y controles relacionan desaceleración variable con IVC en pacientes en labor de parto y consideran que el aumento de tensión en el saco amniótico durante las contracciones uterinas puede comprimir en forma simultánea vena y arterias causando desaceleración.
En conclusión, la desaceleración variable puede presentarse en gestantes sin labor de parto, con membranas íntegras en presencia de IVC.

\section{Declaración de financiamiento y de conflictos de interés:}

Los autores declaran no tener conflictos de interés.

\section{Correspondencia:}

Jorge Díaz Herrera

Loma de las Fresias 125. Surco.

Lima, Perú

Correo electrónico: jorge.diaz@upch.pe

\section{REFERENCIAS BIBLIOGRÁFICAS}

1. Mastrobattista JM, Toy EC. Placenta, cord and membranes. En: Fleischer AC, Toy EC, LeeW, Manning FA, Romero R, editors. Sonography in Obstetrics and Gynecology. New York: McGraw Hill; 2011.p. 155-185.

2. Feldstein VA, Harris RD, Machin GA. Ultrasound evaluation of the placenta and umbilical cord. En: Callen PW, editor. Ultrasonography in obstetrics and gynecology. Philadelphia: Saunders Elsevier; 2008.p. 721-757.

3. Díaz JA, Díaz A. Placenta, cordón umbilical y líquido amniótico. En: Huamán M, Sosa A, Pacheco $\mathrm{J}$, editores. Ecografía en Obstetricia, medicina fetal y ginecología. Lima: Instituto Latinoamericano de Salud Reproductiva y Escuela Peruana de Ultrasonido; 2009.p. 73-81.

4. Clark JFJ. Fetal heart monitoring in detection 
velamentous insertion of the umbilical cord. J Natl Med Assoc. 1984; 76:292-4.

5. Sepúlveda W, Rojas I, Robert Ja, Shcnapp C, Alcalde JL. Prenatal detection of velamentous insertion of the umbilical cord: a prospective color Doppler ultrasound study. Ultrasound Obstet Gynecol. 2003; 21(6):564.

6. Lockwood $\mathrm{CH}$, Russo-Stieglitz K. Velamentous umbilical cord insertion. Up To Date (Internet) 2013. (Citado el 11 de marzo del 2013) Disponible en: http://www.uptodate.com

7. Macones GA, Hankins G, Spong CY, Hauth J, Moore T. The 2008 National Institute of Child
Health and Human Development Workshop Report on Electronic Fetal Monitoring. Obstet Gynecol. 2008; 112(3):661-666.

8. Hasegawa J, Matsuoka R, Ichizuka K, Sekizawa A, Farina A, Okai T. Velamentous cord insertion and atypical variable decelerations with no accelerations. Int J GynaecolObstet. 2005; 90:26-30.

Recibido: 30/04/2013

Aceptado: 13/08/2013 